This is the pre-peer reviewed version of the following article:

Maerkle F, Loeffler F F, Schillo S, Foertsch T, Muenster B, Striffler J, Schirwitz C, Bischoff F R, Breitling F, Nesterov-Mueller A (2014)

High-Density Peptide Arrays with Combinatorial Laser Fusing, Advanced Materials, article first published online: 4 MAR 2014; DOI: 10.1002/adma.201305759

which has been published in final form at http://onlinelibrary.wiley.com/doi/10.1002/adma.201305759/abstract

\title{
Title: High-Density Peptide Arrays with Combinatorial Laser Fusing
}

Frieder Maerkle, Felix F. Loeffler, Sebastian Schillo, Tobias Foertsch, Bastian Muenster, Jakob Striffler, Christopher Schirwitz, F. Ralf Bischoff, Frank Breitling*, and Alexander Nesterov-Mueller*

F. Maerkle, Dr. F. F. Loeffler, S. Schillo, T. Foertsch, B. Muenster, J. Striffler, Dr. C. Schirwitz, Dr. F. Breitling, and Dr. A. Nesterov-Mueller Karlsruhe Institute of Technology, Hermann-von-Helmholtz-Platz 1, 76344 EggensteinLeopoldshafen, Germany

E-mail: alexander.nesterov-mueller@kit.edu, frank.breitling@kit.edu

Dr. F. R. Bischoff

German Cancer Research Center, Im Neuenheimer Feld 280, 69120 Heidelberg, Germany

Keywords: peptide arrays, peptide synthesis, particle patterning, laser processing, immunoassays

Cost-efficient high-density peptide arrays represent an attractive method for high-throughput identification of peptide-protein interactions. They are essential to reduce the consumption of reagents required for the binding assay and, thus, save rare and expensive proteins. The potential of high-density peptide arrays is especially intriguing for peptidome research, e.g. investigating the humoral immune response to viral infections ${ }^{[1,2]}$ or in auto antigen discovery. ${ }^{[3,4]}$ However, today's peptide array market is still dominated by the over 20 year old SPOT synthesis, ${ }^{[5]}$ which provides only low spot densities and which achieves at most a few hundred spots per $\mathrm{cm}^{2}$ if pre-synthesized peptides are spotted. ${ }^{[6]}$ 
Other methods to produce high density arrays are based on lithography. ${ }^{[7-9]}$ Yet, these methods have a principle drawback in comparison to the SPOT synthesis: The necessity of one chemical coupling cycle for each amino acid in a sequential order.

Xerographic approaches for peptide array synthesis require only a single chemical coupling step to accomplish the coupling of all amino acids in one synthesis layer in parallel. In biofunctional xerography, patterns of charged amino acid particles are printed on a functionalized substrate with a custom-designed laser printer according to an electrical charge pattern generated on organic photoconductor drums. Once deposited on the substrate, the particles are heated to initiate the coupling of the amino acids. However, the spot resolution of the laser printer is in the sub millimeter order, ${ }^{[10]}$ which is due to the technical limits of xerography. To further increase the spot density, an advanced method for the production of peptide arrays based on high voltage complementary metal oxide semiconductor (CMOS) chips has been developed. ${ }^{[11,12]}$ However, the maximum spot density in a completely combinatorial synthesis was restricted to 10,000 peptide spots per $\mathrm{cm}^{2}$, which is equivalent to a $100 \mu \mathrm{m}$ spot pitch. ${ }^{[13]}$ Higher spot densities demand significantly higher technical efforts in the CMOS approach, including the development of smaller high voltage transistors, more durable bonding wires, and enhanced surface coatings compatible with the peptide chemistry. The small dimensions of the CMOS chips are an additional drawback, limiting the total number of peptide spots on the array.

We describe a novel method of peptide array synthesis, which combines the high spot densities achieved by light-controlled lithographic methods ${ }^{[7-9]}$ with the cost-efficiency of one-cycle-per-layer coupling in biofunctional xerography. ${ }^{[10,14]}$ Applying combinatorial laser fusing, we demonstrate a peptide array synthesis with a spot density of 40,000 peptide spots per $\mathrm{cm}^{2}$ and feature sizes as small as $10 \mu \mathrm{m}$. 
Each cycle of combinatorial laser fusing is divided into two steps: the physical patterning

(Figure 1) and the chemical coupling (Figure 2).

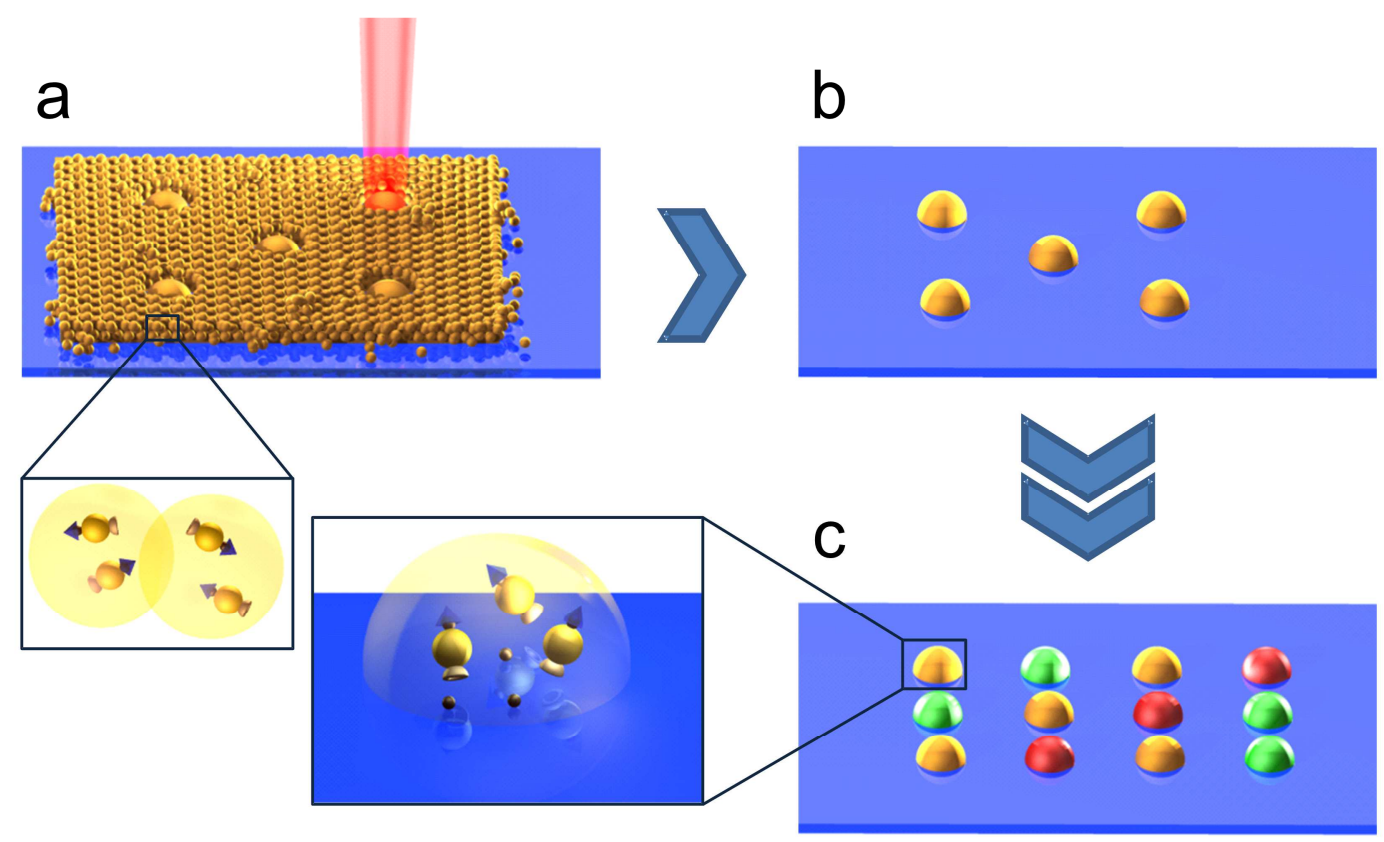

Figure 1. Laser fusing of amino acid patterns. (a) A laser beam fuses micro particles with embedded amino acids to an amino functionalized substrate. (b) Non-fused particles are removed. (c) Repetitive laser fusing with different particles results in a combinatorial amino acid pattern.

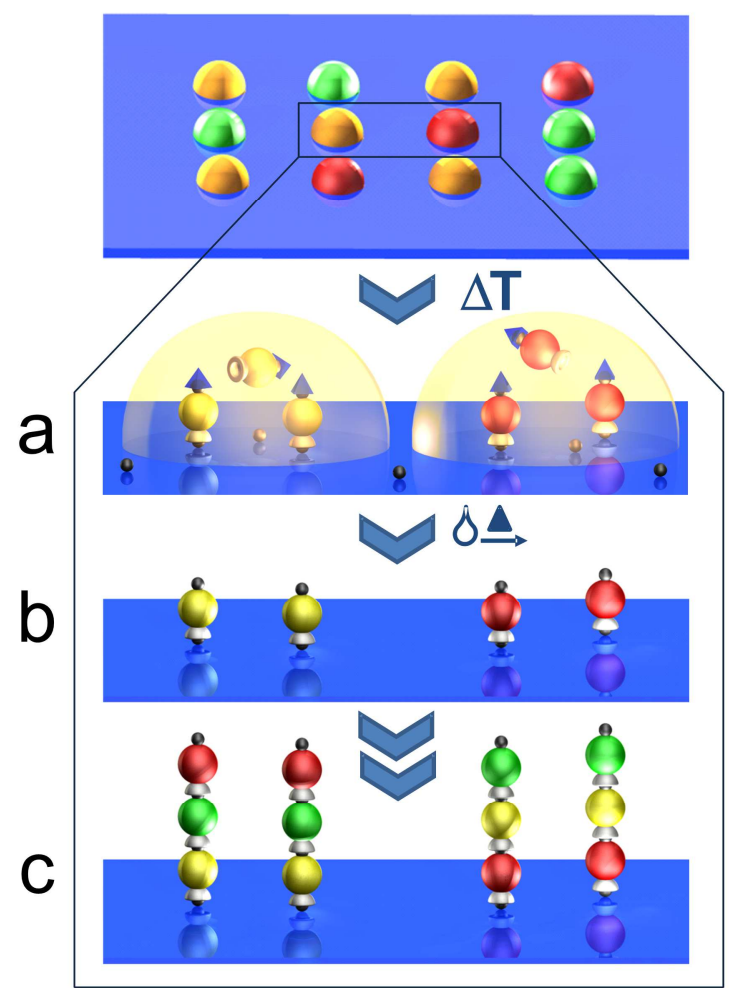


Figure 2. Coupling of combinatorial amino acid pattern. (a) The substrate is heated to allow amino acids to diffuse inside the matrix material and couple to the amino functionalized surface. (b) The matrix material and uncoupled monomers are removed in a washing step. Subsequently, the protecting groups of the monomers are removed to prepare the surface for the next combinatorial patterning. (c) Repetitive combinatorial laser fusing results in a peptide array.

First, we create a combinatorial amino acid spot pattern by fusing different types of micro particles to an amino functionalized glass substrate (Figure 1). The micro particles have a mean size of $2-5 \mu \mathrm{m}$ and are composed of amino acid derivatives embedded in a styreneacrylic copolymer which serves as an inert matrix material (supporting information: monomer particles). As substrate we used glass slides coated with an amino-terminated self-assembled monolayer (SAM). The glass surface was activated and then silanized with 3glycidyloxypropyltrimethoxysilane (3-GPS). This yields an epoxide-terminated SAM. Consecutive epoxide opening with 1,13-diamino-4,7,10-trioxatridecane produces an aminoterminated surface. The preparation of these so-called $\mathrm{AEG}_{3} \mathrm{SAMs}$ is described in detail in the supporting information.

At the beginning of each synthesis cycle, a uniform layer of one micro particle type (i.e. one kind of amino acid) is deposited onto an amino functionalized glass substrate. The particles are deposited from aerosol and form a multilayer of several particle layers. The relative roughness average is below $18 \%$. A detailed description of the process is included in the supporting information. Subsequently, the layer is selectively irradiated with a laser beam (Figure 1a), whereupon the particles within the beam focus are heated and fused to the surface in the form of hemispherical spots. The spot position is determined by the laser beam focus. Afterwards, non-fused particles are removed from the substrate by a jet of air, whereas fused spots remain on the substrate because of their significantly higher adhesion (Figure 1b). By repeating this patterning step with different particle types, we generate the desired 
combinatorial pattern of hemispheres with the corresponding embedded amino acids (Figure 1c).

We observed a significant difference in adhesion force between melted spots and loose particles (supporting information: selective detachment and contamination calculations). We apply an air jet with a velocity of 3-5 $\mathrm{m} \mathrm{s}^{-1}$, at which the drag force selectively detaches loose particles, whereas the melted spots remain on the surface. Even though the melted spots experience a significantly higher adhesion force, the air jet did remove individual melted spots in our experiment. Yet, $96.0 \%$ of the melted spots remained on the surface. To increase the reliability, one can repeat each coating and laser processing an additional time, which then results in an overall success rate of over $99.8 \%$. Due to the non-monodisperse particle size distribution, some of the very small loose particles tend to stick to the surface and cause contaminations. In our experiments, we measured a detachment efficiency of more than $97.7 \%$

In our peptide array synthesis we generated peptide spots of different sizes down to a diameter of $10 \mu \mathrm{m}$, to show that combinatorial laser fusing has, in fact, the potential to produce very high-density peptide arrays with a spot density of up to 1 million spots per $\mathrm{cm}^{2}$. Figure $\mathbf{3 a}$ in supplementary information shows a pattern of two types of particles with different spot sizes of about $10 \mu \mathrm{m}$ and $40 \mu \mathrm{m}$ on a glass slide and Figure 3b a scanning electron microscope image of a fused hemisphere surrounded by unfused particles. The spot size can be adjusted by varying laser intensity and irradiation duration (supporting information: spot morphology). In general, the size of the melted spot depends not only on the laser radiation parameters but also on the thickness of the irradiated particle layer. Assuming the mass conservation in the irradiated volume of the particle layer, it is possible to evaluate the dependency of the minimal fused spot diameter $r_{\min }$ on the thickness of the particle layer $h$ : 
$r_{\min }=\frac{3}{2} \cdot(1-\Pi) \cdot h$

Here, $\Pi$ is the porosity of the particle layer. By varying the laser parameters, we experimentally obtained a minimum spot size of $7 \mu \mathrm{m}$, which is in agreement with the simple geometrical model of equation 1. However, the formation of the hemispheres when fusing the particle layer is, in fact, more complex. It involves the phase transition and the motion of solid and liquid phases. During long irradiation times, we observed melted spots, which accumulated surrounding particle material in a circular manner (Video S1 in supplementary information).
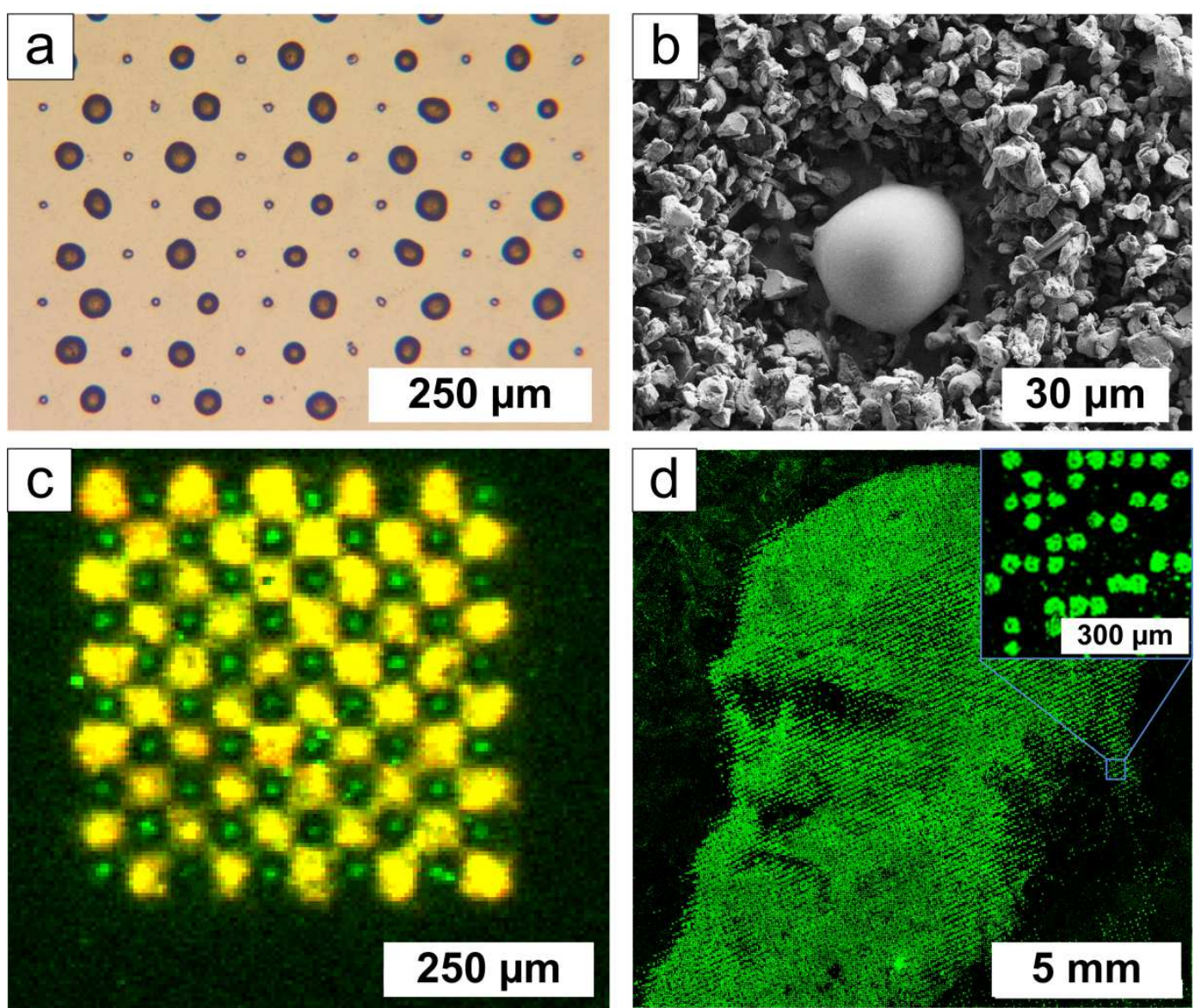

Figure 3. (a) Combinatorial pattern of fused particles on a glass slide composed of the amino acids alanine (small spots) and lysine (large spots). (b) Scanning electron microscope picture of a fused hemispherical spot surrounded by a layer of non-fused amino acid particles. Particles have a mean size of $4.3 \mu \mathrm{m}$. (c) Peptide array with a spot density of 40,000 spots per $\mathrm{cm}^{2}$. The peptides were detected with specific antibodies. The HA peptide was labelled green and the Flag 
peptide was labelled yellow. The feature size of the HA spots is as small as $10 \mu \mathrm{m}$. (d) Molecule array with biotin spots representing the portrait of Charles Darwin detected with fluorescently labelled streptavidin. The image area is $1.5 \times 1.5 \mathrm{~cm}^{2}$ which corresponds to a pattern of 90,000 spots with a spot density of 40,000 spots per $\mathrm{cm}^{2}$.

Although the laser radiation heats and fuses the polymer matrix in form of reaction hemispheres to the surface, the duration of a laser pulse is too short to accomplish the amino acid coupling reaction.

The estimated diffusion coefficient for amino acid derivatives in the melted matrix is $2.7 \times 10^{-14} \mathrm{~m}^{2} / \mathrm{s}$. Thus, the monomers only diffuse in the nanometer scale during the time of irradiation (see supplementary information for an estimation of the diffusion length of the monomers). Therefore, most of the monomers in the melted micrometer sized hemispheres cannot participate in the coupling reaction. As we want to achieve a coupling yield of above $90 \%$, as reported previously in the case of melted toner particles in the xerographic approach, ${ }^{[10]}$ we heat the entire layer of fused reaction hemispheres in an oven. The substrate with the combinatorial spot pattern is kept at $90{ }^{\circ} \mathrm{C}$ for $1 \mathrm{~h}$ under inert gas atmosphere (Figure 2a). Meanwhile, the amino acids, activated as pentafluorophenyl (OPfp) esters, react with the free amino groups on the surface.

After the coupling reaction, the particle matrix and unreacted amino acids are removed in a washing procedure with dimethylformamide (DMF). Unreacted coupling sites are inactivated by routine acylation with $10 \% \mathrm{v} / \mathrm{v}$ acetic anhydride and $20 \% \mathrm{v} / \mathrm{v}$ diisopropylethylamine (DIPEA) in DMF. Subsequently, the Fmoc protecting groups are cleaved from the amino acids using $20 \% \mathrm{v} / \mathrm{v}$ piperidine in DMF to obtain new coupling sites for the next combinatorial synthesis layer (Figure 2b). A detailed protocol for the chemical processing can be found in the supplementary information. Repetitive laser fusing of amino acid particles and coupling of amino acid patterns ultimately yields a high-density peptide array (Figure 2c). 
We repeated the synthesis cycle 9 times, to synthesize the amino acid sequences Tyr-Pro-TyrAsp-Val-Pro-Asp-Tyr-Ala (hemagglutinin- or HA-tag) and Asp-Tyr-Lys-Asp-Asp-Asp-AspLys (Flag-tag) in a chessboard-pattern with a spot-to-spot distance of $50 \mu \mathrm{m}$. This corresponds to a spot density of 40,000 peptide spots per $\mathrm{cm}^{2}$. After the final coupling cycle, we cleaved the side-chain protecting groups from the respective amino acids using trifluoroacetic acid (TFA): tertbutoxycarbonyl (Boc) from Lys, tertbutyl (tBu) from Tyr, and $\mathrm{OtBu}$ from Asp. Subsequently, both peptides were detected with the corresponding fluorescently labelled specific antibodies (Figure 3c).

By extending our method of combinatorial laser fusing, not only peptides, but also other molecules can be synthesized in large-scale high-density array format. The only requirement to synthesize other small molecule arrays is the development of micro particles containing the desired monomers. For instance, we have produced micro particles with embedded biotin derivatives and conducted a high-density patterning with these particles on an amino functionalized glass slide: Figure 3d shows the fluorescent staining of the biotin pattern (for details on staining, see supplementary information).

The successful immunostaining of the peptide array suggests that the laser radiation neither impaired the surface coating required for the peptide synthesis, nor the integrity of the amino acids. To confirm this, we also conducted high-performance liquid chromatography (HPLC) measurements of the embedded amino acids after laser irradiation (supplementary information: HPLC measurements).

In comparison to light controlled lithographic methods for the synthesis of peptide arrays, ${ }^{[8]}$ combinatorial laser fusing allows for equally small spot sizes but requires only a fraction of chemical coupling cycles. During lithographic synthesis, light selectively removes a photolabile transient protecting group at the end of the growing oligomer chains in those areas defined by the lithographic mask. The whole array is then incubated with a solution of 
chemically activated monomers that couple to the deprotected sites. After the coupling reaction, unreacted monomers are washed away. Thus, the principle disadvantage of lithographic synthesis methods is the necessity for one chemical coupling step per monomer in each array layer. In oligonucleotide synthesis, only four different nucleotides are needed. In contrast to this, a combinatorial peptide synthesis requires 20 different amino acids, which results in 300 coupling cycles for the synthesis of an array of 15 -meric peptides $(20 \times 15)$. Such large numbers of chemical steps exponentially increase costs and synthesis artefacts. Combinatorial laser fusing only requires 15 coupling cycles for the synthesis of an array of 15-meric peptides.

The fact that combinatorial laser fusing requires only one chemical cycle to couple all 20 amino acids in a single layer allows for a significant reduction of time for the array synthesis. The time for particle patterning comprises the deposition of the particle layer $(20 \times$ $0.6 \mathrm{~min}=12 \mathrm{~min})$, spot fusing $(10 \mathrm{~ms} / \mathrm{spot})$ and selective particle removal $(20 \times$ $1 \mathrm{~min}=20 \mathrm{~min}$ ). In the case of 500.000 spots per array, which corresponds to a microscope slide with an effective area of $12.5 \mathrm{~cm}^{2}$ and a spot density of 40.000 per $\mathrm{cm}^{2}$, the combinatorial patterning will take $1.4 \mathrm{~h}$. Taking into account the peptide chemistry, i.e. coupling reaction (60 min), capping step (45 min) and removal of the protecting groups (51 $\mathrm{min}$ ), the total time for the synthesis of one layer of an array with 500,000 spots is approx. 4.5 h. In contrast, in lithographical array synthesis, light-induced cleaving of the photo labile protecting groups requires $20 \mathrm{~min}^{[7]}$ Without taking into account the time-consuming chemical washing steps, this already results in $6.7 \mathrm{~h}(20 \times 20 \mathrm{~min})$ per layer.

Our results successfully demonstrate the synthesis of high-density peptide arrays with a spot density of 40,000 spots per $\mathrm{cm}^{2}$ in a combinatorial manner. Feature sizes as small as $10 \mu \mathrm{m}$ show the potential of the method for even higher spot densities. Separation of the physical patterning and the chemical coupling steps are a major advantage, allowing for a significant 
reduction of coupling cycles in comparison to lithographic methods, since only one coupling cycle per layer is necessary instead of one coupling cycle per monomer.

Increasing the spot density in combination with the reduction of synthesis cycles will significantly decrease the price per peptide spot. In the SPOT synthesis, which offers 24 spots per $\mathrm{cm}^{2}$, a single peptide costs $6.50 €$, whereas the xerographic printer renders 800 spots per $\mathrm{cm}^{2}$ for $0.13 €$ per peptide. ${ }^{[6]}$ Following this tendency, we anticipate that our method will allow for the generation of peptide arrays in the microscope slide format offering several hundred thousand or million peptides for a few hundred euros.

The applications of peptide arrays for detecting and characterizing biological interactions are wide and diverse, including for example epitope mapping, enzyme binding, and proteinprotein interactions. ${ }^{[6]}$ All these studies are conducted according to the same principle: Derived from the sequence of a protein of interest, a peptide array in form of overlapping peptides is generated and, then, incubated with the target protein. In several cases it is possible to find distinct binding peptides, which may be used as a basis for the design of inhibiting or activating drugs in protein-protein interactions. A significant lower cost of peptide arrays would enable the search for specific peptide binders without any preliminary information. If we could start with millions of random peptides and stain them with a target protein, we believe that we can always find weak specific binders. In subsequent screenings, the binding can be improved with the introduction of systematic amino acid variations, until strong specific binders are found. Moreover, the efficiency of this approach can probably be increased significantly by introducing synthetic building blocks. With the patterning of biotin, we showed that combinatorial laser fusing can be extended to solid phase coupling of synthetic monomers. 


\section{Supporting Information}

Supporting Information is available from the Wiley Online Library or from the author.

\section{Acknowledgements}

F. Maerkle and Dr. F. F. Loeffler contributed equally to this work.

Dr. F. Breitling, and Dr. A. Nesterov-Mueller contributed equally to this work.

We gratefully acknowledge the financial support of the European Research Council (ERC) for the starting grant "Combinatorial patterning of particles for peptide synthesis", the European Commission (EC) Seventh Framework Programme (FP7) for "TARGETBINDER", and the Carl-Zeiss-Foundation for the postdoc grant.

[1] I. S. Georgiev, N. A. Doria-Rose, T. Q. Zhou, Y. Do Kwon, R. P. Staupe, S. Moquin, G. Y. Chuang, M. K. Louder, S. D. Schmidt, H. R. Altae-Tran, R. T. Bailer, K. McKee, M. Nason, S. O'Dell, G. Ofek, M. Pancera, S. Srivatsan, L. Shapiro, M. Connors, S. A. Migueles, L. Morris, Y. Nishimura, M. A. Martin, J. R. Mascola, P. D. Kwong, Science 2013, 340, 751.

[2] J. F. Scheid, H. Mouquet, N. Feldhahn, M. S. Seaman, K. Velinzon, J. Pietzsch, R. G. Ott, R. M. Anthony, H. Zebroski, A. Hurley, A. Phogat, B. Chakrabarti, Y. X. Li, M. Connors, F. Pereyra, B. D. Walker, H. Wardemann, D. Ho, R. T. Wyatt, J. R. Mascola, J. V. Ravetch, M. C. Nussenzweig, Nature 2009, 458, 636.

[3] H. B. Larman, Z. M. Zhao, U. Laserson, M. Z. Li, A. Ciccia, M. A. M. Gakidis, G. M. Church, S. Kesari, E. M. LeProust, N. L. Solimini, S. J. Elledge, Nat Biotechnol 2011, 29, 535.

[4] W. H. Robinson, L. Steinman, Nat Biotechnol 2011, 29, 500.

[5] R. Frank, Tetrahedron 1992, 48, 9217.

[6] C. Katz, L. Levy-Beladev, S. Rotem-Bamberger, T. Rito, S. G. Rüdiger, A. Friedler, Chemical Society Reviews 2011, 40, 2131.

[7] S. P. Fodor, J. L. Read, M. C. Pirrung, L. Stryer, A. T. Lu, D. Solas, Science 1991, $251,767$.

[8] S. Buus, J. Rockberg, B. Forsstrom, P. Nilsson, M. Uhlen, C. Schafer-Nielsen, Mol Cell Proteomics 2012, 11, 1790.

[9] J. P. Pellois, X. C. Zhou, O. Srivannavit, T. C. Zhou, E. Gulari, X. L. Gao, Nat Biotechnol 2002, 20, 922.

[10] V. Stadler, T. Felgenhauer, M. Beyer, S. Fernandez, K. Leibe, S. Güttler, M. Gröning, K. König, G. Torralba, M. Hausmann, Angewandte Chemie International Edition 2008, 47, 7132.

[11] A. Nesterov, K. König, T. Felgenhauer, V. Lindenstruth, U. Trunk, S. Fernandez, M. Hausmann, F. R. Bischoff, F. Breitling, V. Stadler, Review of Scientific Instruments 2008, 79, 035106.

[12] F. Löffler, J. Wagner, K. König, F. Märkle, S. Fernandez, C. Schirwitz, G. Torralba, M. Hausmann, V. Lindenstruth, F. Bischoff, Aerosol Science and Technology 2011, 45, 65. [13] F. Loeffler, C. Schirwitz, J. Wagner, K. Koenig, F. Maerkle, G. Torralba, M. Hausmann, F. R. Bischoff, A. Nesterov-Mueller, F. Breitling, Advanced Functional Materials 2012. 
[14] M. Beyer, A. Nesterov, I. Block, K. Konig, T. Felgenhauer, S. Fernandez, K. Leibe, G. Torralba, M. Hausmann, U. Trunk, V. Lindenstruth, F. R. Bischoff, V. Stadler, F. Breitling, Science 2007, 318, 1888.

[15] C. Schirwitz, Purification of Peptides in High-Complexity Arrays: A New Method for the Specific Surface Exchange and Purification of Entire Peptide Libraries, Springer, 2013.

[16] A. Nesterov, E. Dörsam, Y.-C. Cheng, C. Schirwitz, F. Märkle, F. Löffler, K. König, V. Stadler, R. Bischoff, F. Breitling, in Small Molecule Microarrays, Springer, 2010, 109.

[17] A. D. Zimon, Adhesion of dust and powder, Consultants Bureau, New York 1982.

[18] K. L. Johnson, J. A. Greenwood, J Colloid Interf Sci 1997, 192, 326.

[19] W. Cheng, P. F. Dunn, R. M. Brach, J Adhesion 2002, 78, 929.

[20] J. N. Israelachvili, Intermolecular and surface forces: revised third edition, Academic press, 2011.

The table of contents entry should be 50-60 words long, and the first phrase should be bold.

Combinatorial laser fusing is a new method to produce high-density peptide arrays with feature sizes as small as $10 \mu \mathrm{m}$. It combines the high spot densities achieved by lithographic methods with the cost-efficiency of biofunctional xerography. The method is also adapted for other small molecules compatible with solid phase synthesis.

Keyword: peptide arrays

F. Maerkle, F. F. Loeffler, S. Schillo, T. Foertsch, B. Muenster, J. Striffler, C. Schirwitz, F. R. Bischoff, F. Breitling*, and A. Nesterov-Mueller*

Title: High-density peptide arrays with combinatorial laser fusing

ToC figure

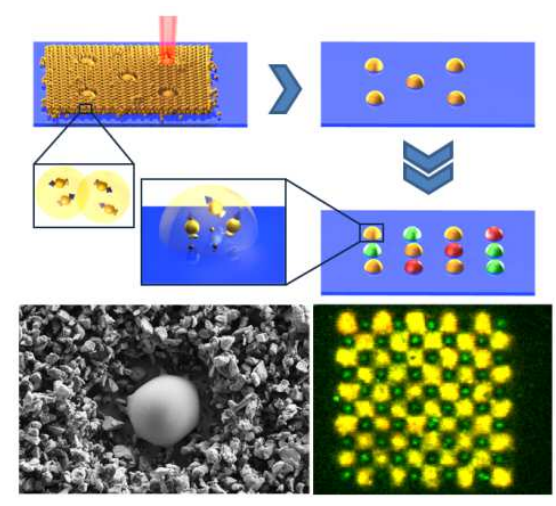


Copyright WILEY-VCH Verlag GmbH \& Co. KGaA, 69469 Weinheim, Germany, 2013.

\section{Supporting Information}

\section{Title: High-Density Peptide Arrays with Combinatorial Laser Fusing}

Frieder Maerkle, Felix F. Loeffler, Sebastian Schillo, Tobias Foertsch, Bastian Muenster, Jakob Striffler, Christopher Schirwitz, F. Ralf Bischoff, Frank Breitling*, and Alexander Nesterov-Mueller*

Monomer particles: The composition of our particles was similar to the composition of particles used in biofunctional xerography. ${ }^{[10]}$ The particles mainly consisted of a styreneacrylic copolymer (88\% w/w) (SLEC PLT-7552 from Sekisui Chemical GmbH) which served as an inert matrix material for the embedded monomers. Only one kind of monomer was embedded in each particle type. For the synthesis of peptide arrays we used pentafluorophenyl (OPfp) activated amino acids with an N-terminal 9fluorenylmethoxycarbonyl (Fmoc) protecting group (10\% w/w). Accordingly, we used biotin-OPfp in the biotin particles. To control the triboelectrical charge of the particles, we added charge additives: sodium-di(aqua)-di(2-hydroxy-3-napthoic acido) ferrate(III) $(0.5 \%$ w/w) and pyrazoloneorange (1.5\% w/w, ABCR GmbH). The size distribution of the particles was measured with the Mastersizer 2000 (Malvern Instruments $\mathrm{GmbH}$ ). The particles had a mean diameter of 3 to $5 \mu \mathrm{m}$, except of the particles containing alanine (Ala) which showed a smaller mean diameter of $2.5 \mu \mathrm{m}$. Figure $\mathbf{S 1}$ shows typical particle size distributions using the example of our particles containing Ala and aspartic acid (Asp). To increase the light absorption of the particles for laser fusing, we added $3 \%(\mathrm{w} / \mathrm{w})$ graphite nano particles. The glass transition temperature of each particle type was measured to be about $70{ }^{\circ} \mathrm{C}$ with differential scanning calorimetry (DSC 204 F1 Phoenix, Netzsch GmbH)

Chemical processing protocol: The surface of our glass support was functionalized with an amino-terminated self-assembled monolayer $\left(\mathrm{AEG}_{3} \mathrm{SAM}\right)$ bearing an (ethylene glycol $)_{3}$ 
spacer. Short EG sequences, starting from 2 EG units, can provide for protein resistance and, thus, prevent unspecific protein adsorption to the surface.

1. Surface silanization: Starting point of each surface coating on the glass substrate is a silanization, which covalently anchors an organic molecule to the silicon dioxide. In this reaction, an ethoxysilane residue was covalently bound to the hydroxyl residues of the surface (Figure S2). The silane serves as the initiator for the subsequent polymerization reaction. First, the glass was irradiated with UV light for $2 \mathrm{~h}$ under atmospheric conditions, using a mercury vapor lamp (TQ 150, Heraeus Noblelight). This treatment removes contaminations and activates the oxide layer by generating surface-bound hydroxyl groups. Then, the slide was incubated in a solution of $2 \mathrm{~mm}$ 2-bromo-N-(3-triethoxysilyl)propyl isobutyramide (bromine silane) and $8 \mathrm{mM} \mathrm{N}$-propyltriethoxysilane (PTES) in anhydrous dichloromethane (DCM) overnight. Subsequently, the DCM was gradually replaced with ethanol (p.a.). The surface was washed three times for $5 \mathrm{~min}$ each with ethanol, twice for $2 \mathrm{~min}$ each with acetone, and then dried in a stream of compressed air. Finally, the slide was placed in a pre-heated oven at $110{ }^{\circ} \mathrm{C}$ for $2 \mathrm{~h}$ to achieve full condensation of the bromine silane with the surface. After cooling to room temperature, the slide was directly coated with the $\mathrm{AEG}_{3}$ or stored at $4{ }^{\circ} \mathrm{C}$ under argon atmosphere.

2. Surface $\mathbf{A E G}_{3}$ coating: The development of the SAM as a functional coating for the peptide synthesis is described by Schirwitz in more detail. ${ }^{[15]}$ A Solution of $30 \mathrm{~mm} 3-$ glycidylpropyl trimethoxy silane (3-GPS) in anhydrous DCM was prepared and added to the activated (UV irradiation) dry glass substrate. The slides were left to react overnight in a desiccator under argon atmosphere. Subsequently, the surfaces were washed three times for 2 min each with DCM. A solution of $20 \%(\mathrm{v} / \mathrm{v})$ 1,13-diamino-4,7,10-trioxa tridecan (DATT) in anhydrous DMF was directly added to the surfaces without drying. The surfaces were allowed to react for $24 \mathrm{~h}$. The samples were washed five times for 5 min each with DMF, 
twice with methanol for $3 \mathrm{~min}$ each, rinsed with acetone, and then dried in a stream of compressed air. $\mathrm{AEG}_{3}$-coated surfaces were stored at $4{ }^{\circ} \mathrm{C}$ under argon atmosphere.

Figure S3 shows a single unit of the self-assembled monolayer structure with the amino terminus and three ethylene glycol subunits $\left(\mathrm{AEG}_{3}\right)$. The terminal amino group eventually functions as the coupling site for the peptide synthesis.

3. Peptide synthesis protocol: After the coating of the glass surface, the amino acid patterning was performed according to the illustrated combinatorial laser fusing principle. After one complete deposition layer is finished, the substrate was heated to $90{ }^{\circ} \mathrm{C}$ under nitrogen atmosphere for $60 \mathrm{~min}$ and the amino acid derivatives couple to the amino terminated substrate as described in Figure S4. Afterwards, the slide was allowed to cool to room temperature. Initiating the washing, the slide is subjected to a mixture of acetic anhydride, N,N-diisopropylethylamine, and N,N-dimethylformamide ( $\left.\mathrm{Ac}_{2} \mathrm{O} / \mathrm{DIPEA} / \mathrm{DMF}\right)$, with the volume ratios $10 \% \mathrm{Ac}_{2} \mathrm{O}, 20 \%$ DIPEA, and $70 \% \mathrm{DMF}$, for five minutes while shaking and afterwards replaced with a fresh mixture and shaking again for 20 min (removal of copolymer matrix and uncoupled amino acids, capping of uncoupled surface amino residues).

Then, the slide was washed with DMF twice for $5 \mathrm{~min}$, and subsequently $5 \mathrm{~min}$ with acetone. Afterwards it was washed again with DMF for $5 \mathrm{~min}$. Now, a mixture of $20 \%$ piperidine in DMF was applied for 30 min, which cleaved the Fmoc amino terminal protecting group from the coupled amino acids. Finally, the slide was washed three times for 5 min with DMF and afterwards twice with methanol $(\mathrm{MeOH})$ for 3 min each.

Together with the combinatorial laser fusing, these steps were repeated until the final length of the peptides was reached.

After coupling and washing of the final amino acid layer, the peptides have to be side chain deprotected. Therefore, the slide was incubated with dichloromethane (DCM) for $30 \mathrm{~min}$. 
Then, to cleave the side chain protecting groups from the amino acids, the slide was washed three times for $30 \mathrm{~min}$ each in a mixture of $51 \% \mathrm{v} / \mathrm{v}$ trifluoroacetic acid (TFA), $3 \% \mathrm{v} / \mathrm{v}$ triisobutylsilane (TiBS, ultrapure, Sigma, USA), $44 \%$ v/v DCM, and $2 \%$ v/v $\mathrm{H}_{2} \mathrm{O}$. Each of these three washing steps was performed with a fresh mixture. Afterwards, the slide was washed twice with DCM for 5 min, to remove any residuals from the previous washing steps. Now, the slide was washed with DMF for 5 min and then incubated for $30 \mathrm{~min}$ with $5 \% \mathrm{v} / \mathrm{v}$ DIPEA in DMF, to neutralize any acidic residues due to the TFA step. Finally, the substrate was washed twice for 5 min each in DMF and subsequently in methanol twice, again for 5 min each. The substrate was dried in an air flow and was then ready for biological application. The protocols can also be found in ${ }^{[15]}$ and ${ }^{[16]}$.

Particle deposition: We used a custom-built aerosol generation system, ${ }^{[13]}$ to deposit the particles on a substrate (Figure S5). The micro particles were siphoned out of a reservoir through an ejector and guided through a polytetrafluoroethylene (PTFE) tube towards the substrate. In the PTFE tube, the particles were triboelectrically charged. The substrate was placed on a metallic plate to which a voltage of $3 \mathrm{kV}$ was applied. The electric field attracted the charged particles and due to self-assembling effects, ${ }^{[11,12]}$ the particles accumulated in a uniform layer of 5 to $30 \mu \mathrm{m}$ thickness, depending on aerosol and particle parameters.

We determined the relative roughness average $\mathrm{R}_{\mathrm{a}} / \mathrm{H}$ of the particle layers to be below $18 \%$ by analyzing the surface with a confocal laser scanning microscope (VK 9700, Keyence). The mean thickness $\mathrm{H}$ of the layer is defined as

$H=\int z(x, y) d S$

and the roughness average $R_{a}$ as 
$R_{a}=\int|z(x, y)-H| d S$

Laser system: We used an Arcturus PixCell II Laser Capture Microdissection system (LCM). The system consisted of an inverse microscope equipped with a laser diode (wavelength $810 \mathrm{~nm}$, max. output power $100 \mathrm{~mW}$, min. spot size $7.5 \mu \mathrm{m}$ ). For combinatorial particle patterning, we irradiated each spot for $10 \mathrm{~ms}$ with an output power of 40 to $80 \mathrm{~mW}$. To position the substrate, we installed an XY table (SCAN IM 120x100, Märzhäuser Wetzlar $\mathrm{GmbH} \& \mathrm{Co}$.) with an accuracy of $\pm 3 \mu \mathrm{m}$ and a repeat accuracy of $<1 \mu \mathrm{m}$. The substrate was moved automatically in respect to the laser beam by means of the XY table and paused at defined positions to selectively fuse the particles with a laser pulse. Repeated and exact processing of the substrate was enabled through positioning marks on the glass slide. The accuracy of the detection of the positioning marks was about $\pm 2 \mu \mathrm{m}$, so the total accuracy of the system was $\pm 3.6 \mu \mathrm{m}$.

Selective detachment and contamination calculations: We observed a significant difference in adhesion force between melted spots and loose particles which can be estimated with the selective detachment factor $\gamma$ :

$\gamma=\frac{v_{\text {det, spot }}}{v_{\text {det }, \text { particle }}}=\frac{r}{r_{c}}$

Here, $v_{\text {det,spot }}$ and $v_{\text {det,particle }}$ are the air velocities needed to detach the melted spots and the loose particles from the surface, $r$ denotes the particle radius and $r_{c}$ is the particle contact radius. Equation $S 3$ states that if the contact radius and the particle radius are equal $(\gamma=1)$, no selective detachment of loose particles is possible. We applied an air jet with a velocity of 3$5 \mathrm{~m} \mathrm{~s}^{-1}$, at which the drag force was within the selective detachment window, so that selective detachment took place at $\gamma \geq 4$. The derivation of equation S3 is based on equation S4-S6. Equation S4 results from the conservation of the particle angular momentum: 
$F_{\mathrm{det}}=F_{n} \frac{r_{c}}{x \cdot r}$

$F_{d e t}$ is the tangential force needed to remove a particle from the surface, $F_{n}$ is the normal force, and $x$ is a dimensionless process constant. According to different sources, $x$ is $4^{[17]}$ or in the range of $1.5-1.75 .^{[18,19]}$

Equation S5 presents the dependency of the detachment force, which has the form of the Stokes drag force acting on a particle with radius $r$ :

$F_{\text {det }}=F_{\text {drag }}=6 \pi \cdot \mu \cdot r \cdot v$

Here, $\mu$ is the dynamic viscosity and $v$ is the air flow velocity. Equation S6 states the linear dependence of the adhesion force $F_{n}$ in our case on the particle radius $r{ }^{[17]}$

$F_{n}=\frac{A}{6 \cdot D^{2}} \cdot r$

$A$ is the Hamaker constant and $D$ is the distance between the particle and the substrate where van der Waals' forces become significant. ${ }^{[20]} A$ and $D$ are the same for the melted spots and for the loose particles. The arithmetical combination of equation S4-S6 results in equation S3. Deriving equation S3, we assumed that the particles and the melted spots are ideally round and that the contact radius of the melted spots is equal to the radius of the base area.

To take into account possible contaminations in form of remaining loose particles after the selective detachment, we measured the detachment coefficient $\alpha=\left(\mathrm{S}_{0}-\mathrm{S}\right) / \mathrm{S}_{0} \cdot 100 \%>97.7 \%$, where $S_{0}$ denotes the total area and $S$ the contaminated area. We determined the detachment coefficient based on microscopy images of the substrates after removal of loose particles. Remaining particles were detected by analyzing the grey values in the images. 
Non-removed loose particles can only cause contaminations if they remain on free areas predefined for melted spots. Particles remaining in the areas between spots are irrelevant for the peptide synthesis. The same applies for loose particles remaining on already melted spots. In this case, the falsely addressed amino acids require significantly more time to diffuse to the reaction sites than the amino acids within the melted spot. This makes the proposed method robust against possible particle contaminations.

Spot morphology: The melted spots tend to form a hemisphere due to the surface tension of the particle matrix material. However, we have observed deviations in the base area of the spots from an ideal circle (Figure 1). To characterize the spot size we therefore chose the equivalent radius $r_{e q}$

$r_{\text {eq }}=\sqrt{S_{\text {spot }} / \pi}$

$S_{\text {spot }}$ represents the base area. Figure S6 shows the dependency of the equivalent radius on the laser power and the pulse duration.

HPLC measurements: HPLC measurements were exemplarily conducted with particles containing Asp-OPfp (Asp = aspartic acid). The particles were deposited on a glass substrate using the aerosol generation system and irradiated with the laser setup (wavelength $810 \mathrm{~nm}$, power $100 \mathrm{~mW}$, duration approx. $1 \mathrm{~s}$, laser focus diameter $7.5 \mu \mathrm{m}$ ). Non-fused particles were removed with a jet of air. Fused particles were dissolved in dioxane and analyzed (C18 column, buffer A: $50 \mathrm{~mm}$ triethylammonium phosphate $\mathrm{pH} 2.25$, buffer B: $10 \%$ buffer A in $90 \%$ acetonitrile). Untreated particles dissolved in dioxane were analyzed as reference (Figure S7). The HPLC analysis showed good stability of the Asp compound in laser fusing.

Diffusion length of monomers: We have estimated the characteristic diffusion length of a monomer $l_{\text {mon }}$ in the melted matrix with the relation 
where $t$ is the characteristic diffusion time and $D$ is the diffusion coefficient calculated according to the Einstein-Smoluchowski relation:

$D=\frac{k_{B} T}{6 \pi \eta a}$

Here, $k_{B}$ is the Boltzmann constant, $T$ is the absolute temperature, $\eta$ is the dynamic viscosity, and $a$ is the mean radius of the moving sphere. Taking $\eta=10 \mathrm{~kg} / \mathrm{ms}, a=1 \mathrm{~nm}$, and $T=365 \mathrm{~K}$, we get for the diffusion coefficient $D \approx 2.67 \times 10^{-14} \mathrm{~m}^{2} / \mathrm{s}$. The relatively large radius of the monomer results from the fact that the amino acid derivatives have an Fmoc protecting group and an OPfp ester. Thus, $l_{\text {mon }} \approx 23 \mathrm{~nm}$ for a laser pulse duration of $t=10 \mathrm{~ms}$ and $l_{\text {mon }} \approx 14 \mu \mathrm{m}$ when heating the melted spots in an oven for $60 \mathrm{~min}$.

Immunostaining: After successful peptide synthesis and side chain deprotection with TFA, the peptide arrays were incubated for $10 \mathrm{~min}$ in phosphate buffer saline (PBS-T) with $0.05 \%$ v/v polysorbate 20 (Tween 20, Sigma, USA) and afterwards blocked for $1 \mathrm{~h}$ in blocking buffer for fluorescent western blotting (Rockland Immunochemicals, Inc., USA), to prevent unspecific antibody binding. After one short washing step (10 s) with PBS-T, the arrays were then incubated with monoclonal Mouse Anti-Flag M2 IgG1 (Sigma, USA) antibodies diluted 1:1000 in PBS-T $+1: 10$ blocking buffer. Afterwards, the array was stained with secondary Anti-Mouse antibodies conjugated with DyLight 680 (KPL, Inc., USA). Then, we incubated the peptide array with monoclonal Mouse Anti-HA 12CA5 IgG antibodies (provided by Dr. G. Moldenhauer, DKFZ), diluted 1:1000 in PBS-T $+1: 10$ blocking buffer, and stained with secondary Alexa Fluor 488 Goat Anti-Mouse antibodies (Molecular Probes/Invitrogen, USA). We scanned the peptide array using the excitation wavelengths $532 \mathrm{~nm}$ and $633 \mathrm{~nm}$ with a 
two-color Agilent Scanner G2505C with $5 \mu \mathrm{m}$ resolution and automatically adjusted photomultiplier tube voltages.

The biotin pattern was prepared in analogy to the peptide array staining, but stained with a streptavidin Alexa Fluor 546 conjugate 1:5000 in PBS-T and scanned with a GenePix 4000B scanner with a resolution of $5 \mu \mathrm{m}$ using the excitation wavelength $532 \mathrm{~nm}$. 


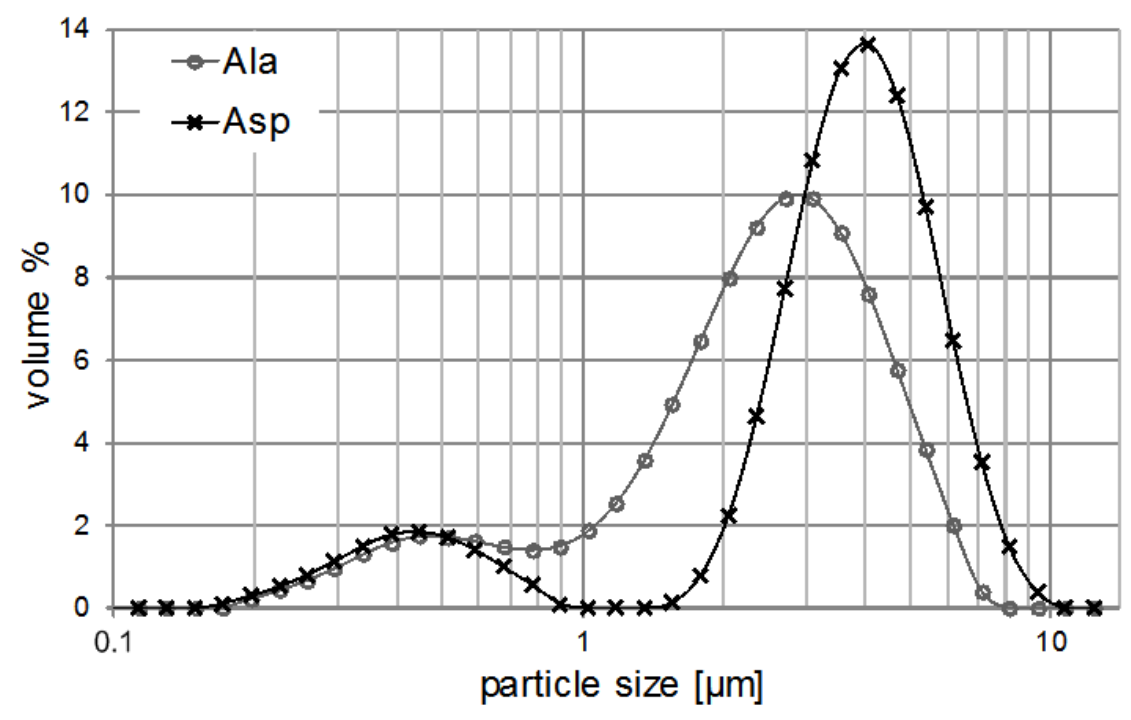

Figure S1. Particle size distribution of micro particles containing Ala (mean size $2.5 \mu \mathrm{m}$ ) and Asp (mean size $3.7 \mu \mathrm{m}$ ) measured by laser diffraction (Malvern Mastersizer 2000, Malvern Instruments).

a<smiles>CCOCCCNC(=O)C(C)(C)Br</smiles>
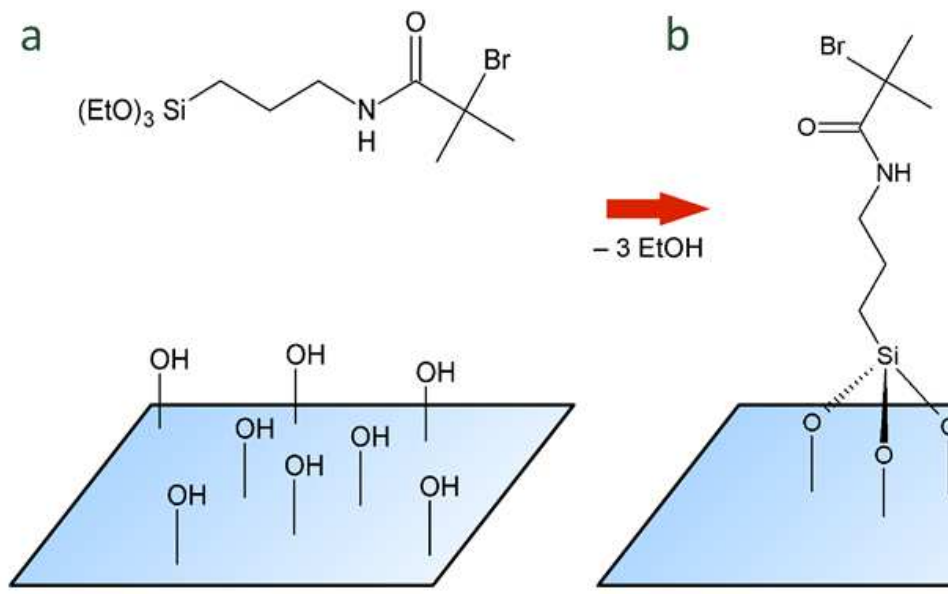

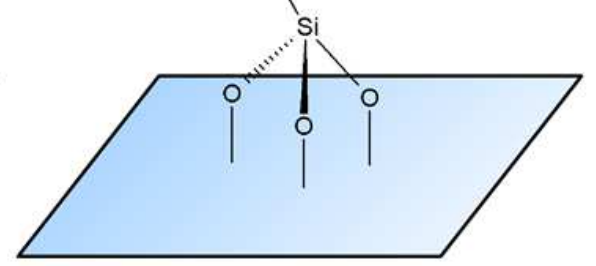

Figure S2. Bromine silane and the substrate surface. (a) exhibiting hydroxyl residues, (b) the bromine silane couples to the surface, releasing ethanol. 


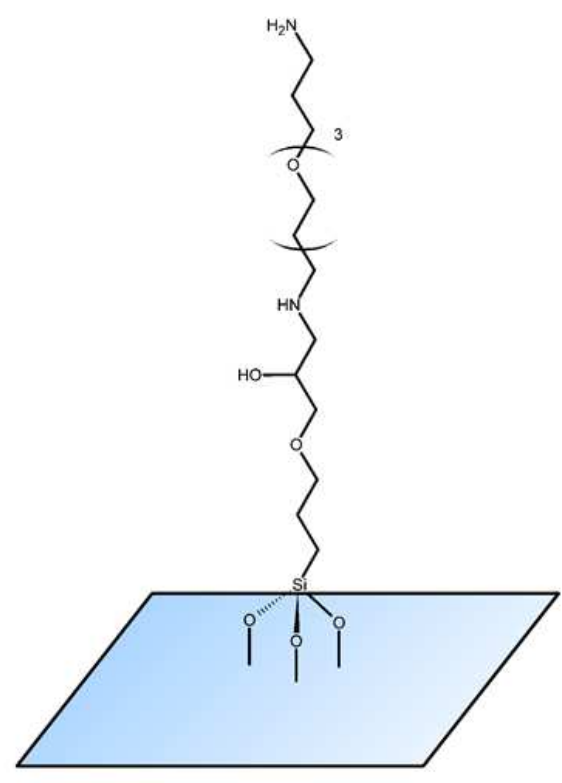

Figure S3. Single unit of the self-assembled monolayer structure with the amino terminus and three ethylene glycol units $\left(\mathrm{AEG}_{3}\right)$. The amino group eventually functions as the coupling site for the peptide synthesis.
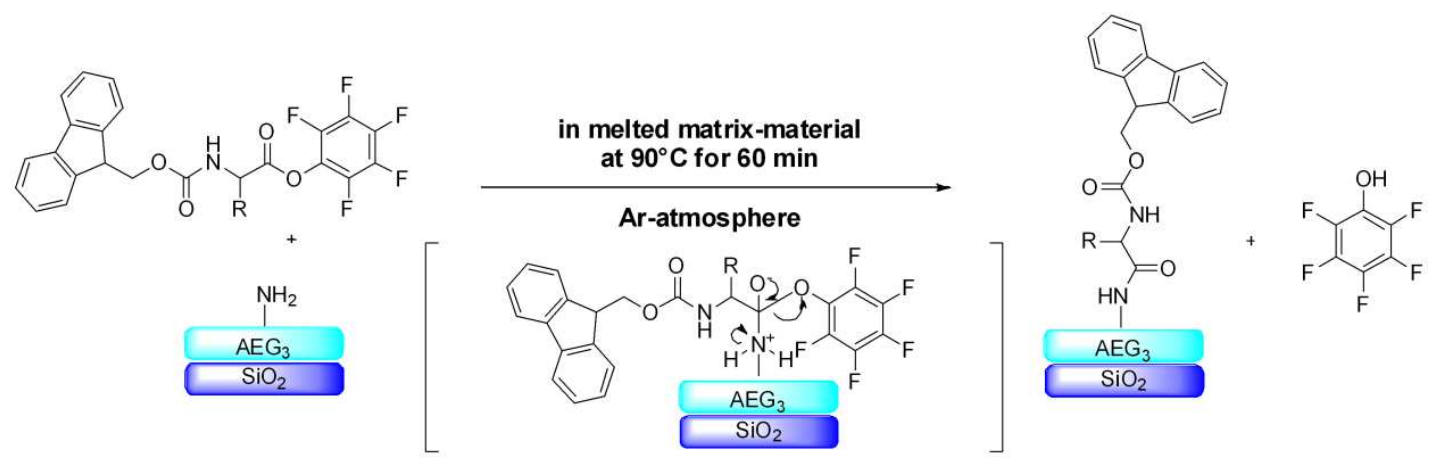

Figure S4. Condensation reaction of an Fmoc protected amino acid derivative, activated as OPfp ester, with the amino-terminated $\mathrm{AEG}_{3}$ surface. 


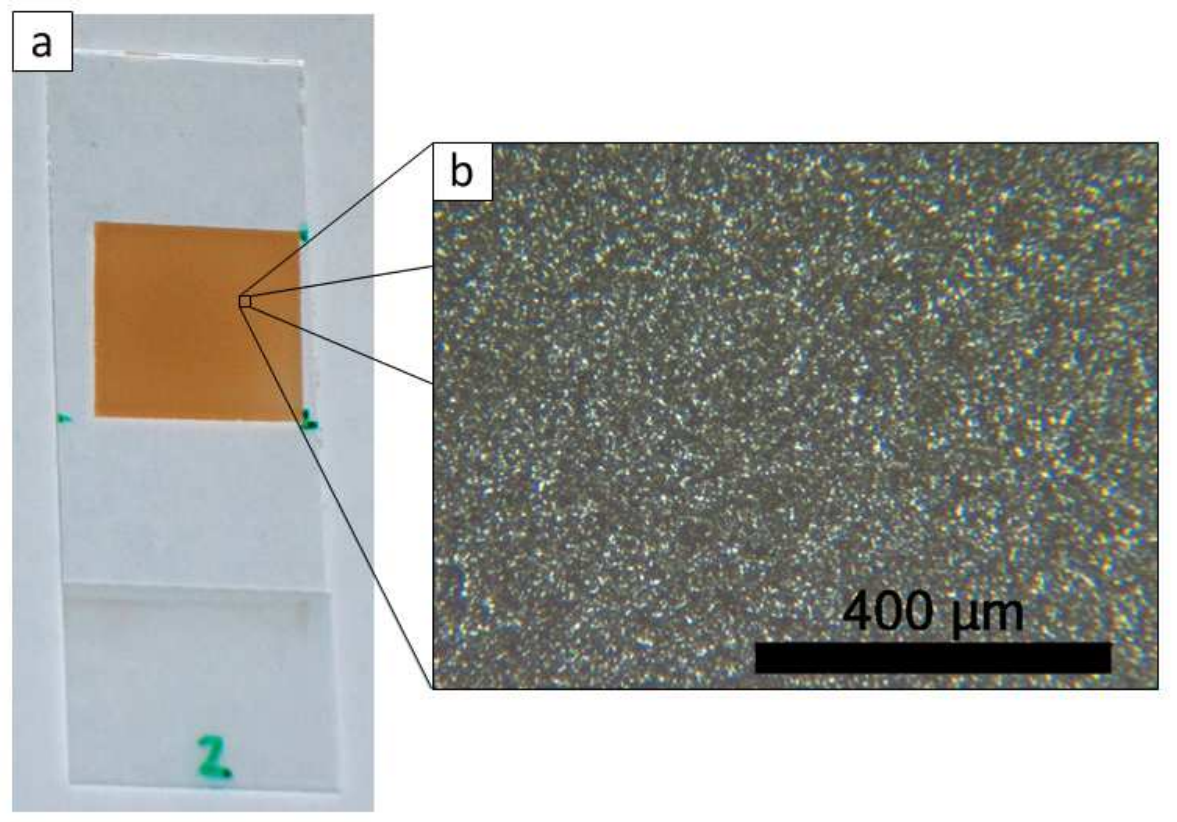

Figure S5. Glass slide coated with amino acid particles from aerosol, particles contain asparagine, mean particle size is $4.0 \mu \mathrm{m}$. (a) Particle coated microscope slide, approx. $0.475 \mathrm{mg} / \mathrm{cm}^{2}$. (b) Image of the particle layer trough an optical microscope. 


\section{a}

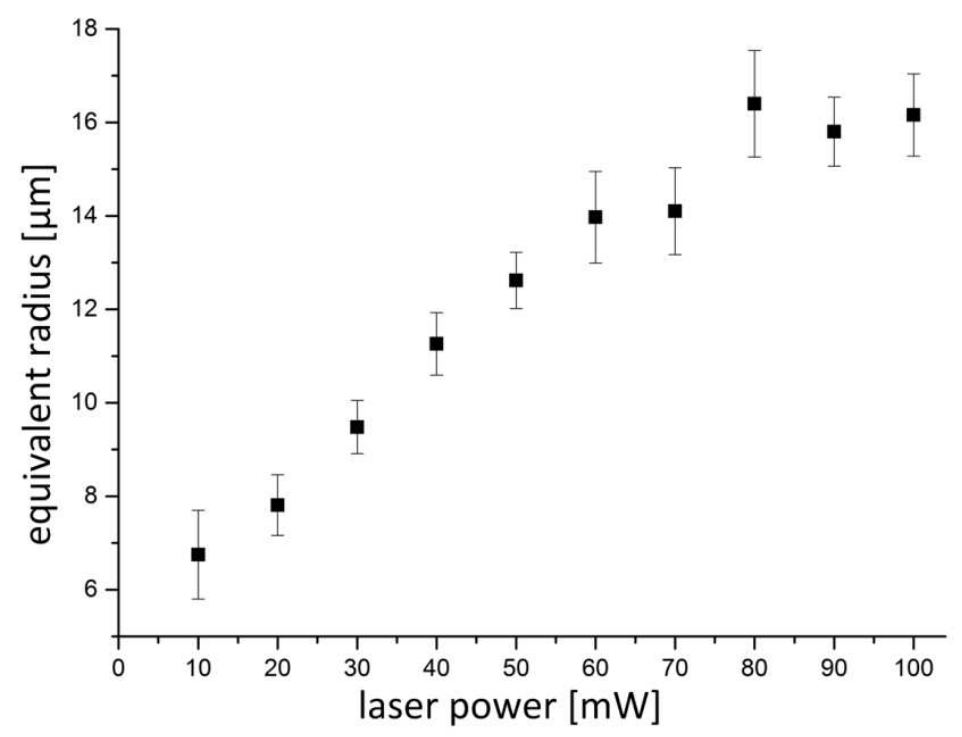

\section{b}

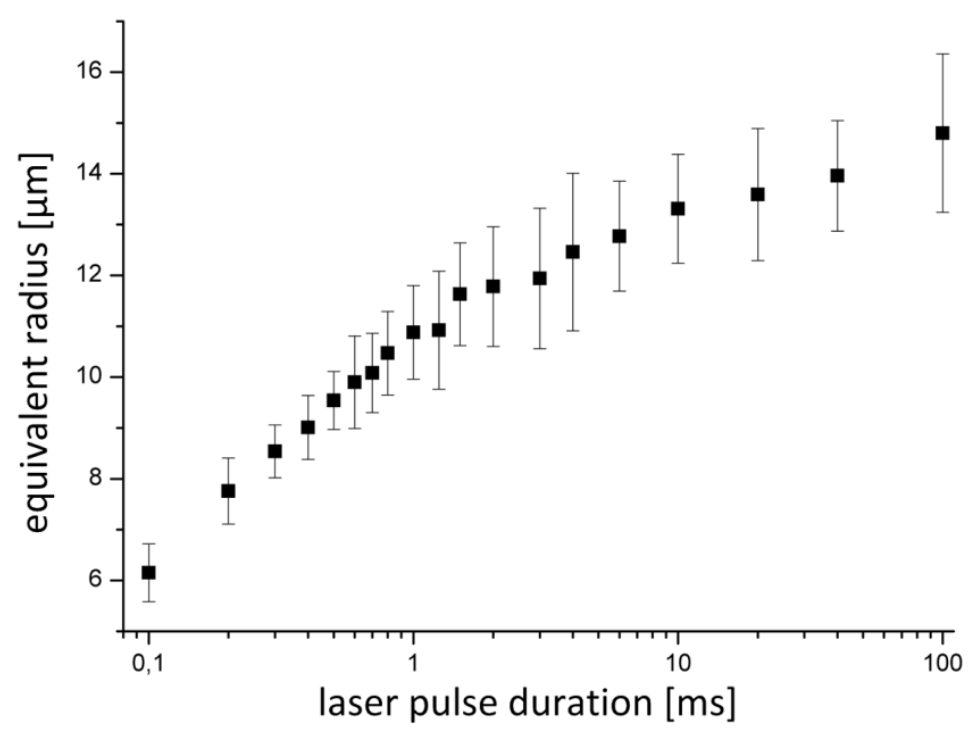

Figure S6. Influence of laser parameters on the equivalent radius of the melted spots, laser wavelength $810 \mathrm{~nm}$, laser focus diameter $7.5 \mu \mathrm{m}$, particles containing alanine, median particle size $2.5 \mu \mathrm{m}$. (a) Fixed laser pulse duration $500 \mu \mathrm{s}$, varying laser power 10-100 mW. (b) Fixed laser power $25 \mathrm{~mW}$, varying laser pulse duration $0.1-100 \mathrm{~ms}$. 

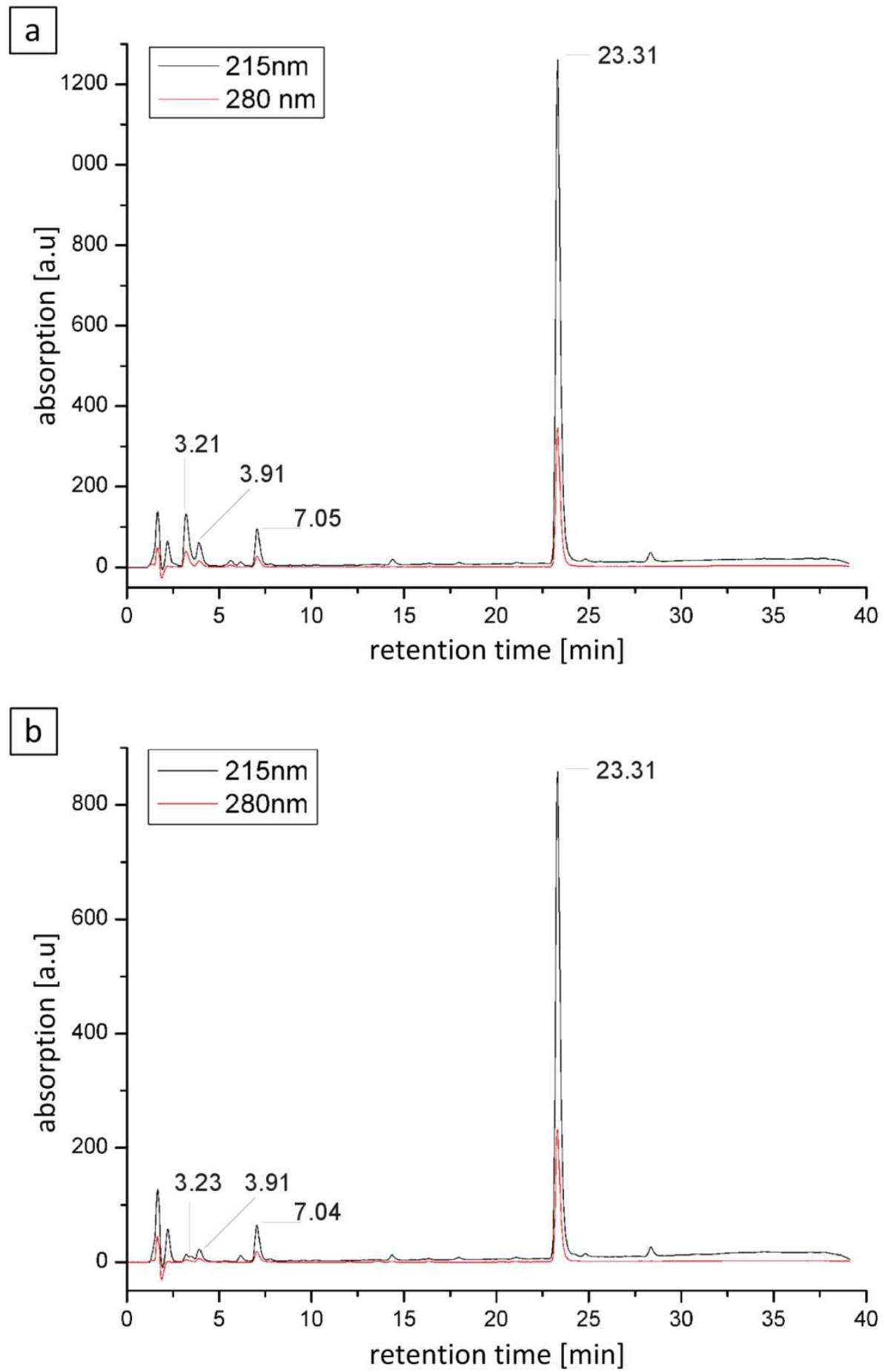

Figure S7. HPLC measurements of micro particles containing aspartic acid dissolved in dioxane. (b) Particles irradiated with a laser. (a) Reference with untreated particles. 
Video S1. The Video shows the behavior of a fused hemisphere irradiated with repetitive laser pulses. The melted material "ingests" particles from the surrounding area in a circular manner. The laser power was $30 \mathrm{~mW}$, the laser pulse duration $10 \mathrm{~ms}$, the pulse frequency $4.8 \mathrm{~Hz}$ and the laser focus diameter $7.5 \mu \mathrm{m}$. 